Supporting Information

\title{
Iron-facilitated transformation of mesoporous spinel nanosheets to oxyhydroxide active species in oxygen evolution reaction
}

\author{
Yue Li, ${ }^{a}$ Xinxuan Lin ${ }^{a}$ and Jing Du $* a, b$
}

a. State Key Laboratory of Applied Organic Chemistry, Key Laboratory of Advanced Catalysis of Gansu Province, College of Chemistry and Chemical Engineering, Lanzhou University, Lanzhou 730000, China. Email: dujing@1zu.edu.cn

b. Key Laboratory of Advanced Energy Materials Chemistry (Ministry of Education), Nankai University, Tianjin 300071, China
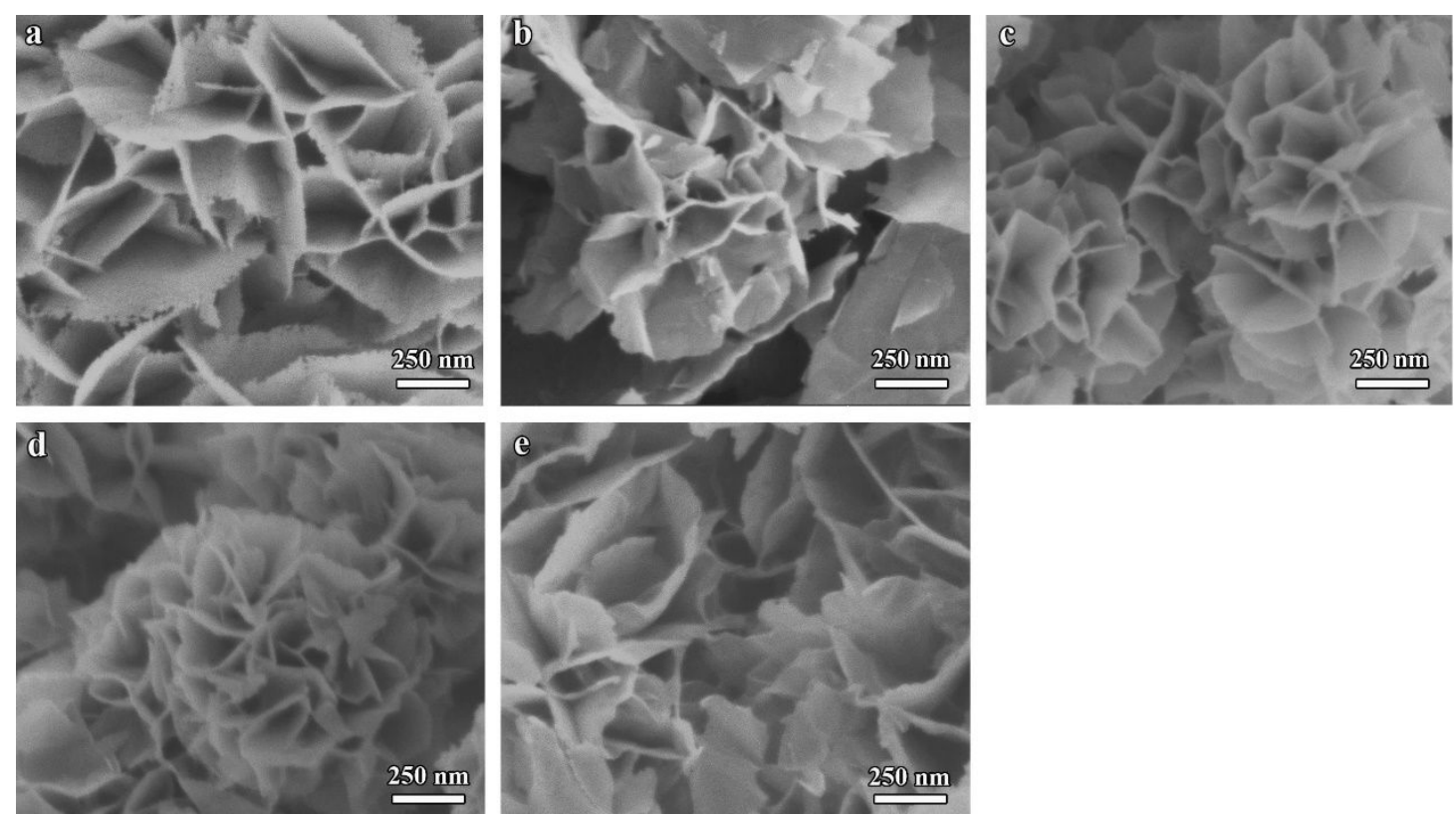

Figure S1. SEM images of as-prepared $\mathrm{Fe}_{\mathrm{x}} \mathrm{Ni}_{1-\mathrm{x}} \mathrm{Co}_{2} \mathrm{O}_{4}$ oxides. (a) $\mathrm{NiCo}_{2} \mathrm{O}_{4}$, (b) $\mathrm{Fe}_{0.1} \mathrm{Ni}_{0.9} \mathrm{Co}_{2} \mathrm{O}_{4}$, (c) $\mathrm{Fe}_{0.2} \mathrm{Ni}_{0.8} \mathrm{Co}_{2} \mathrm{O}_{4}$, (d) $\mathrm{Fe}_{0.3} \mathrm{Ni}_{0.7} \mathrm{Co}_{2} \mathrm{O}_{4}$, (e) $\mathrm{Fe}_{0.4} \mathrm{Ni}_{0.6} \mathrm{Co}_{2} \mathrm{O}_{4}$. 

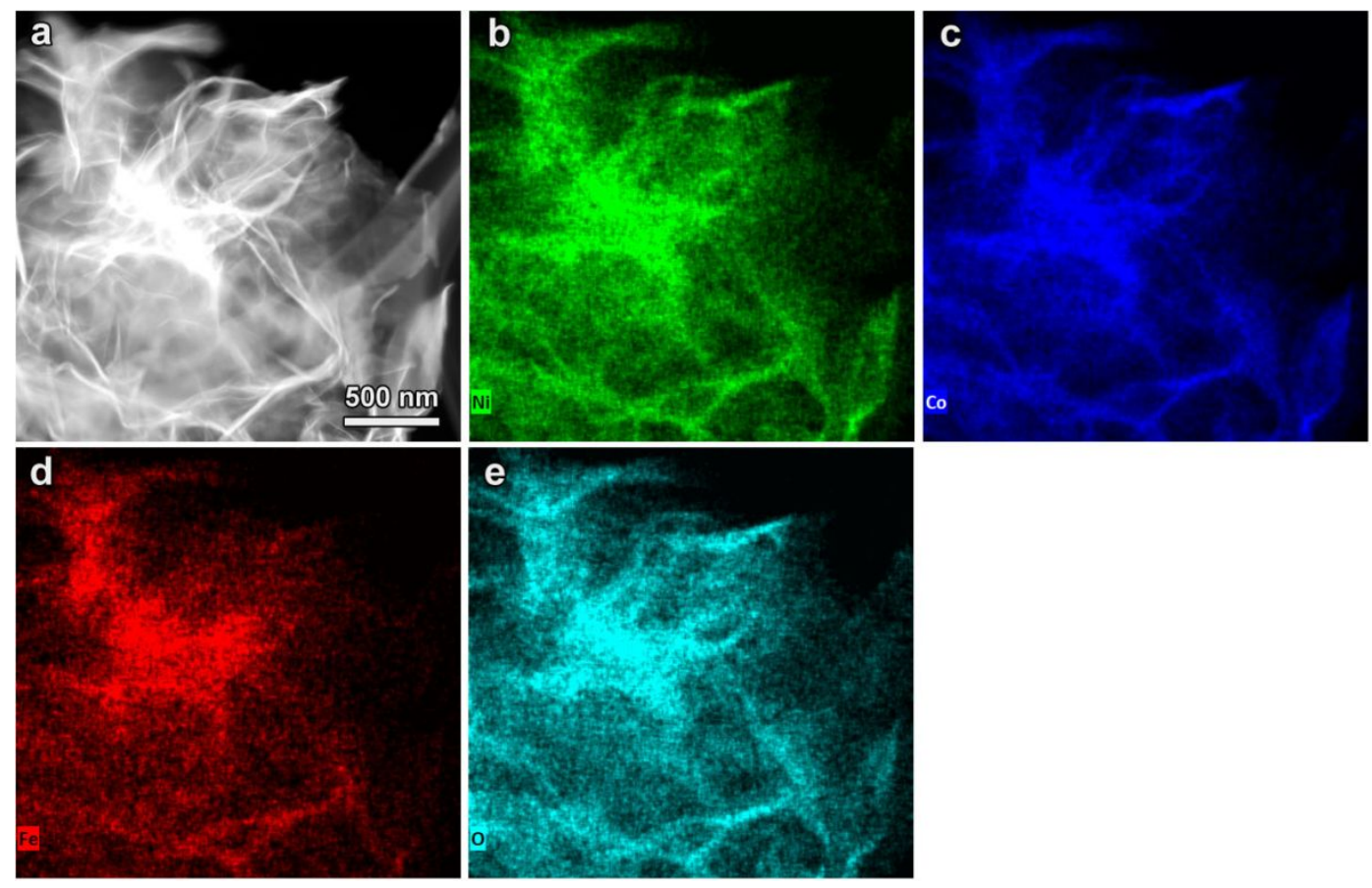

Figure S2. Elements mapping of the $\mathrm{Fe}_{0.2} \mathrm{Ni}_{0.8} \mathrm{Co}_{2} \mathrm{O}_{4}$ nanosheets. (a) HADDF image, (b) Ni, (c) Co, (d) Fe and (e) O element. 


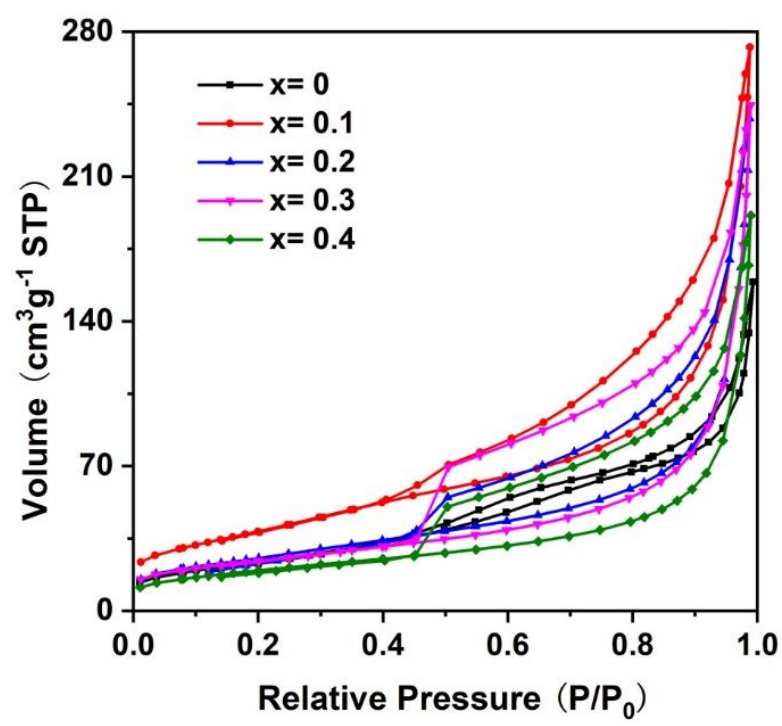

Figure S3. $\mathrm{N}_{2}$ adsorption-desorption isotherms of as-prepared $\mathrm{Fe}_{\mathrm{x}} \mathrm{Ni}_{1-\mathrm{x}} \mathrm{Co}_{2} \mathrm{O}_{4}$ oxides. 


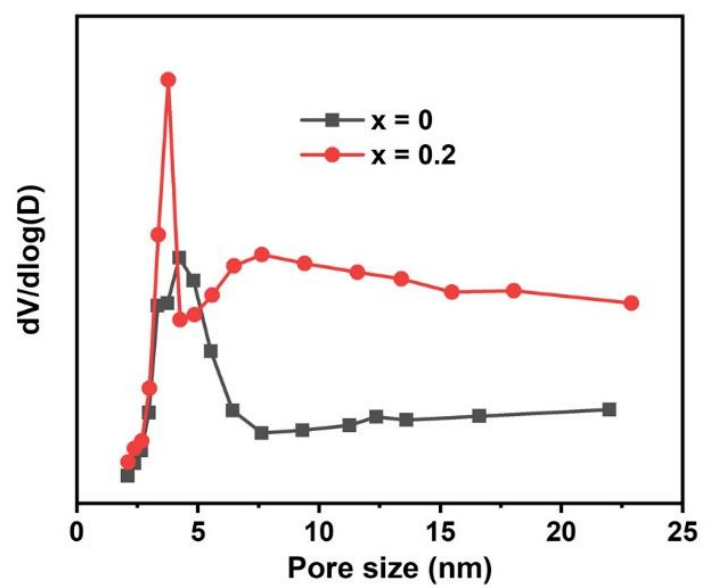

Figure S4. Pore size distribution diagrams of pure $\mathrm{NiCo}_{2} \mathrm{O}_{4}$ and $\mathrm{Fe}_{0.2} \mathrm{Ni}_{0.8} \mathrm{Co}_{2} \mathrm{O}_{4}$. 

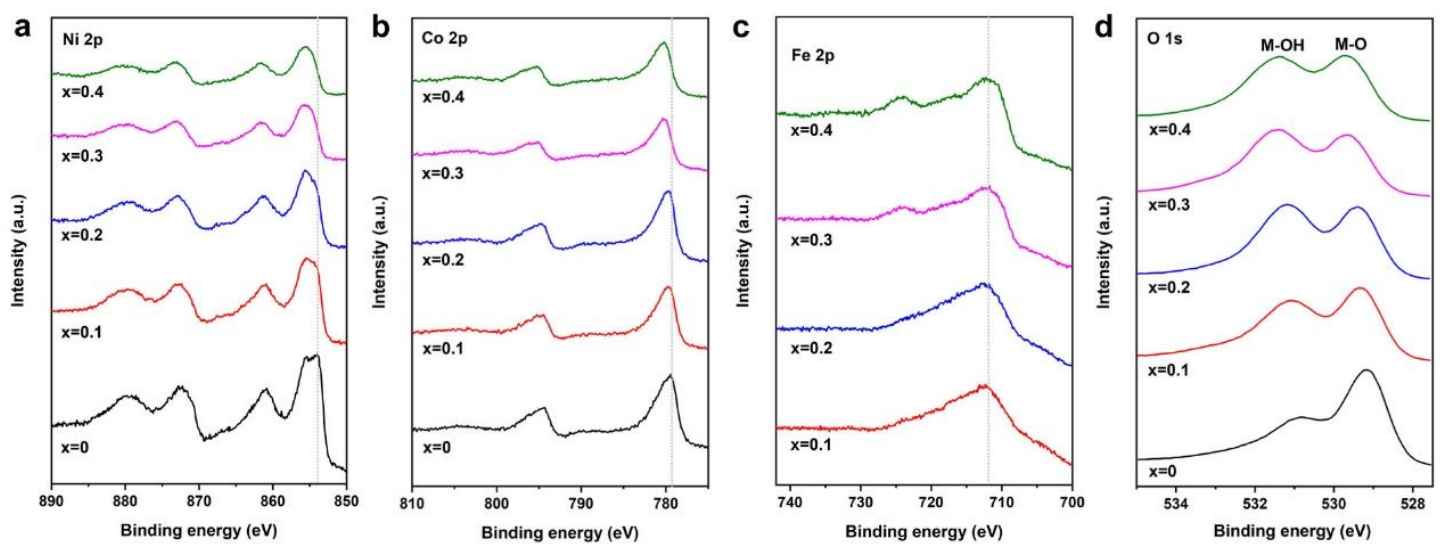

Figure S5. (a) Ni 2p, (b) Co 2p, (c) Fe 2p and (d) O 1s XPS spectra for the $\mathrm{Fe}_{\mathrm{x}} \mathrm{Ni}_{1-}$ ${ }_{x} \mathrm{Co}_{2} \mathrm{O}_{4}$ samples. The $\mathrm{Ni}$ and $\mathrm{Co}$ peaks shift toward higher binding energy with increasing iron content, which indicates that Fe substitution leads to the increased oxidation state of $\mathrm{Ni}$ and decreased oxidation state of $\mathrm{Co}$. The iron peaks almost remain constant. The intensity of metal-OH peaks also increases with increasing iron content. 


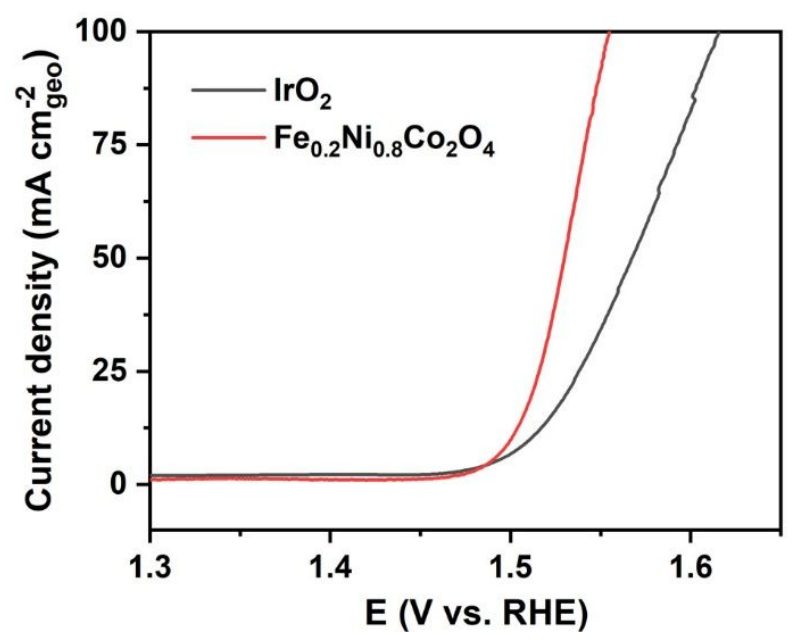

Figure S6. Linear-sweep voltammograms of $\mathrm{Fe}_{0.2} \mathrm{Ni}_{0.8} \mathrm{Co}_{2} \mathrm{O}_{4}$ nanosheets and commercial $\mathrm{IrO}_{2}$ in $1 \mathrm{M} \mathrm{KOH}$ with a scan rate of $5 \mathrm{mV} \mathrm{s}^{-1}$. 

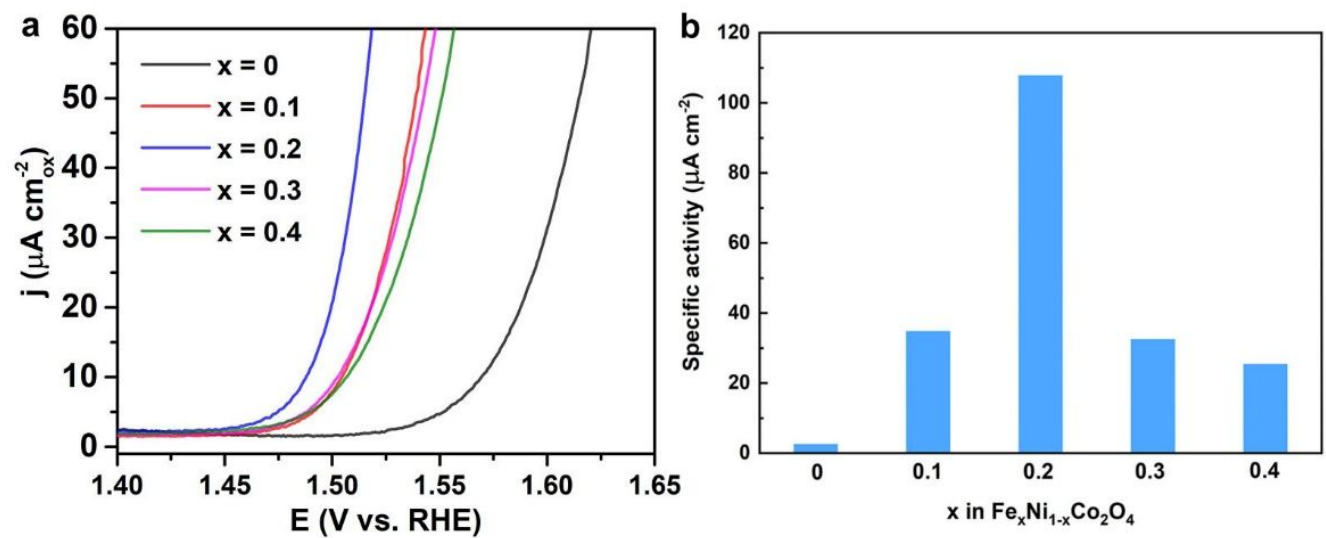

Figure S7. (a) Linear-sweep voltammograms normalized to the surface area (determined by Brunauer-Emmett-Teller measurements) of $\mathrm{Fe}_{\mathrm{x}} \mathrm{Ni}_{1-\mathrm{x}} \mathrm{Co}_{2} \mathrm{O}_{4}$ nanosheets $(\mathrm{x}=0,0.1,0.2,0.3$, and 0.4$)$ in $1 \mathrm{M} \mathrm{KOH}$ with a scan rate of $5 \mathrm{mV} \mathrm{s}^{-1}$. (b) specific activity of $\mathrm{Fe}_{\mathrm{x}} \mathrm{Ni}_{1-\mathrm{x}} \mathrm{Co}_{2} \mathrm{O}_{4}$ nanosheets at $1.53 \mathrm{~V}$ versus RHE. 

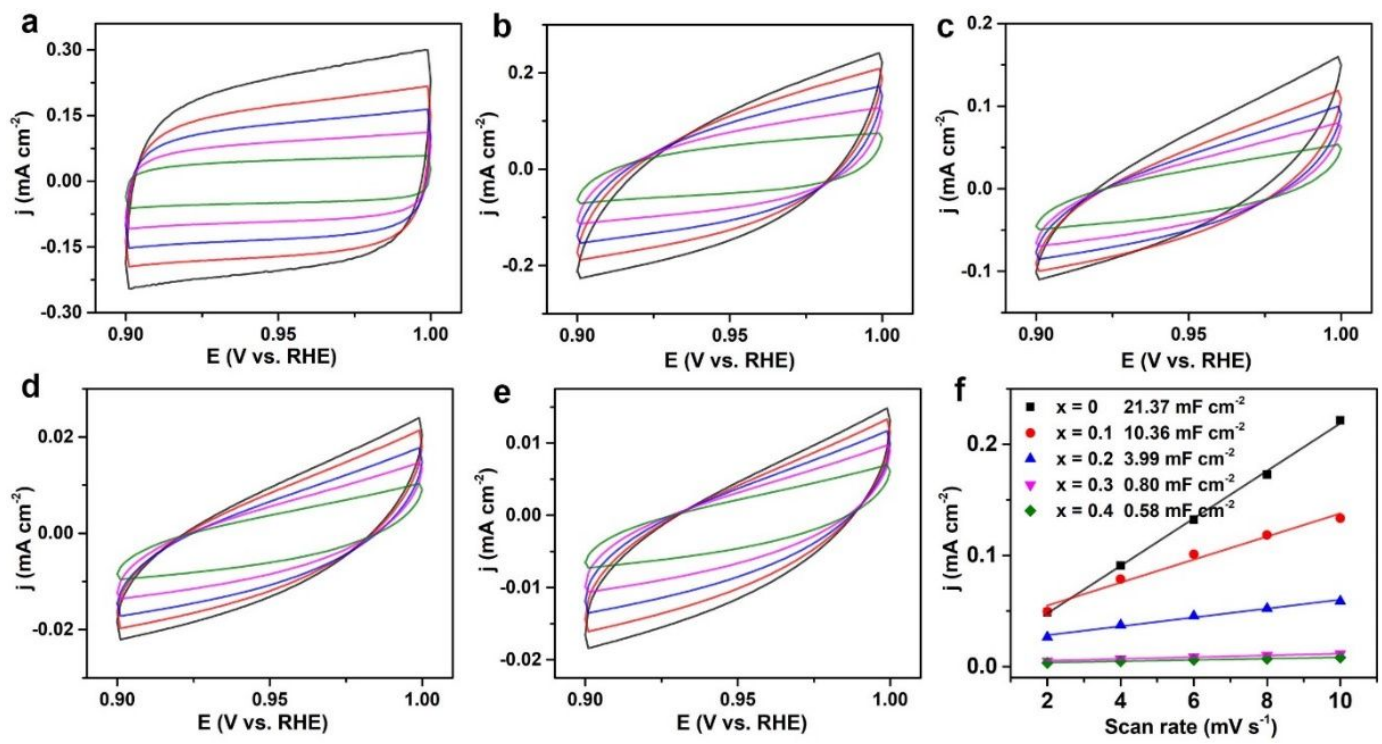

Figure S8. Cyclic voltammogram curves of (a) $\mathrm{NiCo}_{2} \mathrm{O}_{4}$, (b) $\mathrm{Fe}_{0.1} \mathrm{Ni}_{0.9} \mathrm{Co}_{2} \mathrm{O}_{4}$, (c) $\mathrm{Fe}_{0.2} \mathrm{Ni}_{0.8} \mathrm{Co}_{2} \mathrm{O}_{4}$, (d) $\mathrm{Fe}_{0.3} \mathrm{Ni}_{0.7} \mathrm{Co}_{2} \mathrm{O}_{4}$, (e) $\mathrm{Fe}_{0.4} \mathrm{Ni}_{0.6} \mathrm{Co}_{2} \mathrm{O}_{4}$ in $1 \mathrm{M} \mathrm{KOH}$ with different scan rate. (f) Fitting results for the electrochemical double layer capacitance $\left(\mathrm{C}_{\mathrm{dl}}\right)$ data. 

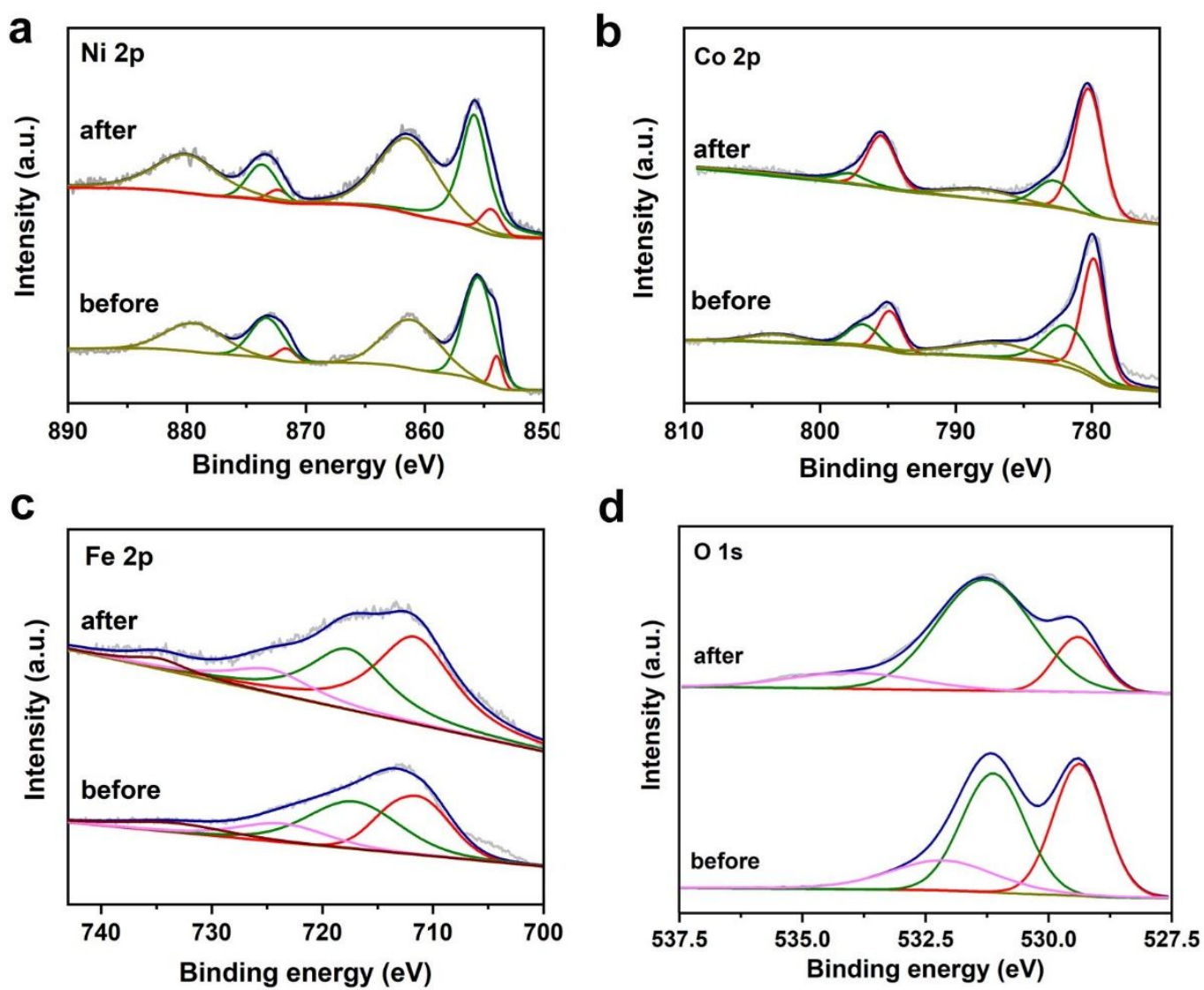

Figure S9. (a) Ni 2p, (b) Co 2p, (c) Fe 2p and (d) O 1s XPS spectra before and after OER durability test for the $\mathrm{Fe}_{0.2} \mathrm{Ni}_{0.8} \mathrm{Co}_{2} \mathrm{O}_{4}$ nanosheets. 
Table S1. The atom ratios of $\mathrm{Ni} / \mathrm{Fe}$ in all prepared oxides obtained from ICP.

\begin{tabular}{cc}
\hline Sample & $\mathrm{Ni} / \mathrm{Fe}$ \\
\hline $\mathrm{NiCo}_{2} \mathrm{O}_{4}$ & $1 / 0$ \\
$\mathrm{Fe}_{0.1} \mathrm{Ni}_{0.9} \mathrm{Co}_{2} \mathrm{O}_{4}$ & $7.8 / 1$ \\
$\mathrm{Fe}_{0.2} \mathrm{Ni}_{0.8} \mathrm{Co}_{2} \mathrm{O}_{4}$ & $3.4 / 1$ \\
$\mathrm{Fe}_{0.3} \mathrm{Ni}_{0.7} \mathrm{Co}_{2} \mathrm{O}_{4}$ & $2.0 / 1$ \\
$\mathrm{Fe}_{0.4} \mathrm{Ni}_{0.6} \mathrm{Co}_{2} \mathrm{O}_{4}$ & $1.3 / 1$ \\
\hline
\end{tabular}


Table S2. The catalytic performance comparison of recently reported $\mathrm{NiCo}_{2} \mathrm{O}_{4}$-based OER catalysts.

\begin{tabular}{|c|c|c|c|c|}
\hline Electrocatalyst & Substrate & $\begin{array}{c}\eta @ 10 \mathrm{~mA} \\
\mathrm{~cm}^{-2}(\mathrm{mV})\end{array}$ & $\begin{array}{l}\text { Tafel slope } \\
\left.(\mathrm{mV} \mathrm{dec})^{-1}\right)\end{array}$ & Ref. \\
\hline $\begin{array}{c}\text { Mesoporous } \\
\mathrm{Fe}_{0.2} \mathrm{Ni}_{0.8} \mathrm{Co}_{2} \mathrm{O}_{4} \\
\text { nanosheets }\end{array}$ & glassy carbon & 270 & 39 & $\begin{array}{l}\text { This } \\
\text { work }\end{array}$ \\
\hline $\mathrm{MoS}_{2} / \mathrm{rFe}-\mathrm{NiCo}_{2} \mathrm{O}_{4}$ & carbon paper & $270 \pm 3$ & 39 & {$[1]$} \\
\hline $\begin{array}{c}\mathrm{NiCo}_{2-\mathrm{x}} \mathrm{Fe}_{\mathrm{x}} \mathrm{O} 4 \\
\text { nanoboxes }\end{array}$ & carbon paper & 274 & 42 & {$[2]$} \\
\hline \multirow{2}{*}{$\begin{array}{c}\mathrm{NiCo}_{2.148} \mathrm{O}_{4} \\
\text { mesoporous } \\
\text { nanosheets }\end{array}$} & Ni Foam & 190 & 50 & \multirow{2}{*}[3]{} \\
\hline & glassy carbon & 290 & 40 & \\
\hline $\begin{array}{c}\mathrm{Co}_{3} \mathrm{O}_{4} / \mathrm{NiCo}_{2} \mathrm{O}_{4} \\
\text { double-shelled } \\
\text { nanocages }\end{array}$ & Ni Foam & 340 & 88 & {$[4]$} \\
\hline $\begin{array}{c}\mathrm{NiCo}_{2} \mathrm{O}_{4} \text { nanorod } \\
\text { arrays }\end{array}$ & Ti sheet & $\begin{array}{c}320 \\
\left(25 \mathrm{~mA} \mathrm{~cm}^{-2}\right)\end{array}$ & 118 & {$[5]$} \\
\hline $\mathrm{NiCo}_{2} \mathrm{O}_{4} / \mathrm{S}-\mathrm{rGO}$ & glassy carbon & 280 & 57 & {$[6]$} \\
\hline $\begin{array}{c}\mathrm{NiCo}_{2} \mathrm{O}_{4} \\
\text { nanoframe }\end{array}$ & glassy carbon & 265 & 82 & [7] \\
\hline $\begin{array}{c}\text { Nitrogen-doped } \\
\text { carbon coated } \\
\mathrm{NiCo}_{2} \mathrm{O}_{4} \text { nanorods }\end{array}$ & glassy carbon & 296 & 53 & [8] \\
\hline $\begin{array}{l}\text { phosphorous-doped } \\
\qquad \mathrm{NiCo}_{2} \mathrm{O}_{4}\end{array}$ & Ni Foam & 300 & 120 & [9] \\
\hline $\mathrm{CePO}_{4} / \mathrm{NiCo}_{2} \mathrm{O}_{4}$ & Ni Foam & $\begin{array}{c}281 \\
\left(20 \mathrm{~mA} \mathrm{~cm}^{-2}\right)\end{array}$ & 74 & {$[10]$} \\
\hline
\end{tabular}




\section{Reference}

1. J. Li, D. Chu, H. Dong, D. R. Baker and R. Jiang, J. Am. Chem. Soc., 2020, 142, $50-54$.

2. Y. Huang, S. L. Zhang, X. F. Lu, Z.-P. Wu, D. Luan and X.-W. Lou, Angew. Chem., Int. Ed., 2021, 60, 11841-11846.

3. J. Yin, J. Jin, H. Liu, B. Huang, M. Lu, J. Li, H. Liu, H. Zhang, Y. Peng, P. Xi and C.-H. Yan, Adv. Mater., 2020, 32, 2001651.

4. H. Hu, B. Guan, B. Xia and X. W. Lou, J. Am. Chem. Soc., 2015, 137, 5590-5595.

5. T. Zhou, C. Wang, Y. Shi, Y. Liang, Y. Yu and B. Zhang, J. Mater. Chem. A, 2020, 8, 1631-1635.

6. Rebekah, C. Viswanathan and N. Ponpandian, Nanoscale Adv., 2021, 3, 3216-3231. 7. Z. Chen, B. Zhao, Y.-C. He, H.-R. Wen, X.-Z. Fu, R. Sun and C.-P. Wong, Mater. Chem. Front., 2018, 2, 1155-1164.

8. M. Ahmad, B. Xi, Y. Gu and S. Xiong, Inorg. Chem. Front., 2021, 8, 3740-3747.

9. W. Chu, Z. Shi, Y. Hou, D. Ma, X. Bai, Y. Gao and N. Yang, ACS Appl. Mater. Interface, 2020, 12, 2763-2772.

10. W. Gao, W. Gou, Y. Ma, R. Wei, J. C. Ho and Y. Qu, ACS Appl. Energy Mater., $2019,2,5769-5776$. 